\title{
The possibility of existence of massive pulsars with quark core
}

\section{Partha Roy Chowdhury ${ }^{1} \dagger$}

$\dagger$ Department of Physics, Chandrakona Vidyasagar Mahavidyalaya, Vidyasagar University, Paschim Medinipur, West Bengal -721201, India

Department of Physics, Govt. Degree College Kamalpur, Tripura University, Tripura-799285, India

E-mail: royc.partha@gmail.com

\begin{abstract}
Existence of ultra-high densities at the core makes neutron stars superb astrophysical laboratories for a wide range of physical studies [1-3]. Recent observations [4, 5] on massive neutron stars powerfully influence the high-density equation of state because of the existence of the neutron star maximum mass. Depending on the model, the energy density of quark matter can be lower than that of the nucleonic EoS at higher densities, implying the possibility of transition to quark matter inside the core. In comparison with recent observations [4,5] i.e. $1.93 \mathrm{M}_{\odot} \leq \mathrm{M} \leq 2.05 \mathrm{M}_{\odot}$, a pure nucleonic EoS without quarks and the EoS including quarks determine that the maximum masses of NS rotating with frequency $v \sim 667 \mathrm{~Hz}$ below r-mode instability are $\sim 1.95 \mathrm{M}_{\odot}$ and $\sim 1.72 \mathrm{M}_{\odot}$ respectively. This work suggests that EoS must stiffen at densities exceeding $2-3 \rho_{0}\left(\rho_{0} \sim\right.$ saturation density) to allow the existence of neutron stars having twice solar mass. The existence of deconfined quarks in neutron star interiors is not confirmed with present work. We need to shed more light on the possible existence of exotic matter [6,7] such as deconfined quarks, kaon condensates, and hyperons inside the core of neutron star.
\end{abstract}

XIII Nuclei in the Cosmos

7-11 July, 2014

Debrecen, Hungary

\footnotetext{
${ }^{1}$ Speaker

$\dagger$ Present Address
} 


\section{Introduction}

Various states of matter have been suggested to exist in the ultra-dense centers of neutron stars [1-3]. Some calculations emphasize on the role of quark deconfinement in neutron stars and predict the possible existence of compact stars made of absolutely stable strange quark matter. The question of whether or not quark deconfinement may occur in neutron stars is a topic of interest for construction of equation of state (EoS). In this context, the mass-radius (M$\mathrm{R})$ relationship of neutron stars is of prime importance to understand the high-density lowtemperature regime of the hadronic equation of state (EoS) including or excluding quarks at the stellar core. Depending on this relationship, certain models for the hadronic EoS can either be confirmed or ruled out. Several attempts have been made on measuring the radii and masses [4, 5] of NSs to constrain the uncertainties in the high density behavior of the EoS.

We present a systematic study of the properties of pure hadronic and hybrid compact stars. The nuclear EoS for $\beta$-equilibrated neutron star (NS) matter obtained using a density-dependent effective nucleon-nucleon DDM3Y interaction satisfies the constraints from the observed flow data from heavy-ion collisions. The incompressibility $\mathrm{K}_{\infty}$ for the symmetric nuclear matter (SNM), nuclear symmetry energy $\mathrm{E}_{\text {sym }}\left(\rho_{0}\right)$ at saturation density $\rho_{0}$, the isospin dependent part $\mathrm{K}_{\tau}$ of the isobaric incompressibility and the slope $\mathrm{L}$ are all in excellent agreement with the experimental and observational data.

Depending on the model, the energy density of quark matter can be lower than that of this nuclear EoS at higher densities, implying the possibility of transition to quark matter inside the core and the transition density depends on the particular quark matter model used. We solve the Einstein field equations for rotating stars using pure nuclear matter and quark core. The $\beta$ equilibrated neutron star matter, along with different EoS's for crust, can support largest mass measurements.

\section{Compact Stars with and without Quark}

If rapidly rotating compact stars were nonaxisymmetric, they would emit gravitational waves in a very short time scale and settle down to axisymmetric configurations. Therefore, we need to solve for rotating and axisymmetric configurations in the framework of general relativity. Treating the matter as a perfect fluid the energy-momentum tensor $\mathrm{T}^{\mu v}$ is given by

$T^{\mu v}=(\varepsilon+P) u^{\mu} u^{v}-g^{\mu v} P$

where $\varepsilon, \mathrm{P}, \mathrm{u}^{\mu}$ and $\mathrm{g}^{\mu v}$ are the energy density, pressure, four velocity and the metric tensor, respectively. To study the rotating stars the following metric is used

$d s^{2}=-e^{(\gamma+\rho)} d t^{2}+e^{2 \alpha}\left(d r^{2}+r^{2} d \theta^{2}\right)+e^{(\gamma-\rho)} r^{2} \sin ^{2} \theta(d \varphi-\omega d t)^{2}$ 
where the gravitational potentials $\gamma, \rho, \alpha$ and $\omega$ are functions of polar coordinates $r$ and $\theta$ only. The Einstein's field equations for the three potentials $\gamma, \rho$ and $\alpha$ have been solved using the Green's-function technique and the fourth potential $\omega$ has been determined from other potentials. All the physical quantities may then be determined from these potentials. Obviously, at the zero frequency limit corresponding to the static solutions of the Einstein's field equations for spheres of fluid, the present formalism yields the results for the solution of the TolmanOppenheimer-Volkoff (TOV) equation. We use the 'rns' code [8] for calculating the compact star properties which requires EoS in the form of energy density versus pressure ( $\varepsilon$ vs. P) along with corresponding enthalpy and baryon number density and since we are using various EoS for different regions, these are smoothly joined.

The nuclear matter EoS is calculated [9] using the isoscalar and the isovector components of M3Y interaction along with density dependence. The density dependence of the effective interaction, DDM3Y, is completely determined from nuclear matter calculations. The equilibrium density of the nuclear matter is determined by minimizing the energy per nucleon ( $\epsilon$ $=\mathrm{E} / \mathrm{A})$. In a Fermi gas model of interacting neutrons and protons, the $\epsilon$ for isospin asymmetric nuclear matter is the sum of the kinetic part and nuclear potential part calculated using the volume integral of the isoscalar and isovector components of M3Y interaction. The details of the present methodology may be obtained in Ref. [9].

We numerically determine the EoS for the $\beta$-equilibrated charge neutral neutron star matter in which the $\beta$-equilibrated proton fraction is determined from the knowledge of the symmetry energy. Then this nucleonic EoS with thin crust is used to solve the Einstein's field equations by Green's function technique to determine the mass-radius relationship of neutron stars with and without quark cores. The calculations for rotating and static compact stars without quark core are performed using the crustal EoS for thin crust upto number density of $\rho \sim 0.05 \mathrm{fm}^{-3}$ or, density $\xi \approx 7.7 \times 10^{13} \mathrm{~g} / \mathrm{cm}^{3}=0.31 \xi_{0}$ (nuclear saturation density $\xi_{0}=2.5 \times 10^{14} \mathrm{~g} / \mathrm{cm}^{3}$ ) and $\beta$ equilibrated NS matter for stellar core up to $\rho \approx 1.4 \mathrm{fm}^{-3}$ or equivalently up to $\xi \approx 3.5 \times 10^{15}$ $\mathrm{g} / \mathrm{cm}^{3}=14 \xi_{0}$.

The energy density of the quark matter [10] is lower than that of the present EoS for the $\beta$-equilibrated charge neutral NS matter at densities higher than $0.405 \mathrm{fm}^{-3}$ for bag constant $\mathrm{B}^{1 / 4}$ $=110 \mathrm{MeV}[11]$ implying the possible existence of quark core. So we employ the crust EoS up to $\rho \approx 0.05 \mathrm{fm}^{-3}$, the nucleonic EoS up to $\rho \approx 0.4 \mathrm{fm}^{-3}$ or $\xi \approx 7 \times 10^{14} \mathrm{~g} / \mathrm{cm}^{3}=2.8 \xi_{0}$ and finally quark EoS [10] up to $\rho \approx 1.37 \mathrm{fm}^{-3}$ or $\xi \approx 3 \times 10^{15} \mathrm{~g} / \mathrm{cm}^{3}=12 \xi_{0}$ for the calculations of masses and radii for rotating and static neutron star with both nucleons and quark matter inside. The pressures of the present EoS for the $\beta$-equilibrated charge neutral NS matter and the quark matter EoS for bag constant $\mathrm{B}^{1 / 4}=110 \mathrm{MeV}$ are shown in fig. 1 as functions of energy densities. 


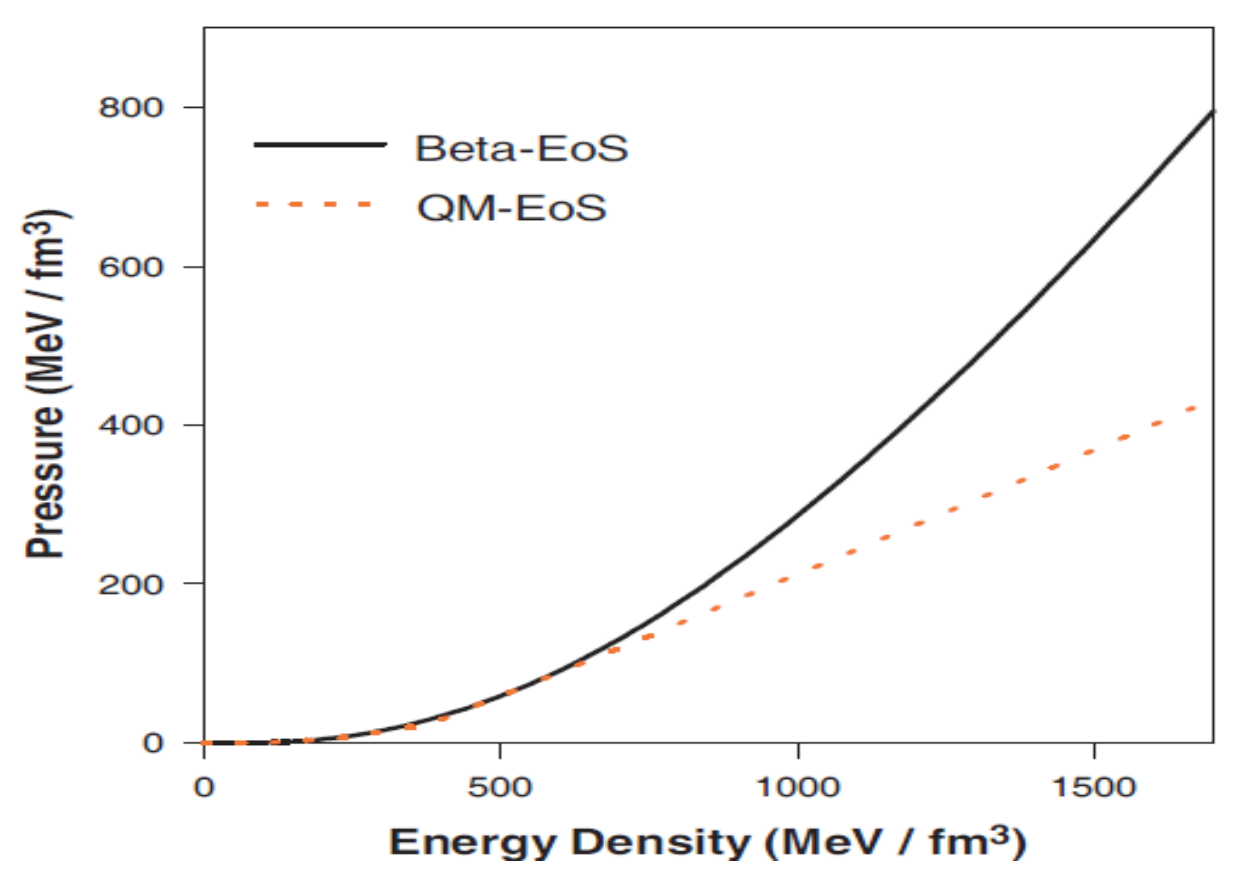

Fig.1 Pressure versus energy density ( $P$ vs. $\varepsilon$ ) plots of $\beta$-equilibrated charge neutral neutron star matter and quark matter.

The results of the calculations for stars with pure nucleonic core [12] without quark and hybrid stars with mixture of quark and nucleons in the stellar core are shown by four M-R curves denoted by 'PRC' in fig.2. Employing the EoSs described in different theoretical approaches [13, 14], various M-R curves are also shown in fig.2 for comparison. The EoSs by theoretical models e.g. AP1, AP4, SQM1, SQM2, PAL1, PAL6, PS, SLY4, ENG, GM3, GS2, MPA1, PCL2, WFF3 listed in fig2. obtained from Ref. $[13,14]$ are primarily nonrelativistic potential models, relativistic field theoretical models and relativistic Dirac-Brueckner-Hartree-Fock models. Since the nature of strong interactions in dense matter is not precisely known yet, the composition of a NS predicted by different theoretical approaches is different. Therefore none of the models is accurate in all aspects. It is clear from fig.2 that NSs with pure nuclear matter inside, the maximum mass for the static case (i.e. time period $\mathrm{T} \sim 0 \mathrm{~ms}$ ) is $1.92 \mathrm{M} \odot$ with radius $\sim$ $9.7 \mathrm{~km}$ and for the star rotating with maximum frequency $v \sim 667 \mathrm{~Hz}$ (i.e. time period $\mathrm{T} \sim 1.5 \mathrm{~ms}$ ) limited by the r-mode instability, the maximum mass turns out to be $1.95 \mathrm{M}_{\odot}$ with radius about 9.9 kilometers. However, the maximum masses for hybrid neutron star with quark core are significantly reduced for both static and rotating stars. The maximum mass of static hybrid star is only $1.68 \mathrm{M}_{\odot}$ with radius about $10.4 \mathrm{~km}$. The maximum mass is increased to $1.72 \mathrm{M}_{\odot}$ with radius about $10.7 \mathrm{~km}$ for the hybrid star rotating with time period $\mathrm{T} \sim 1.5 \mathrm{~ms}$. 


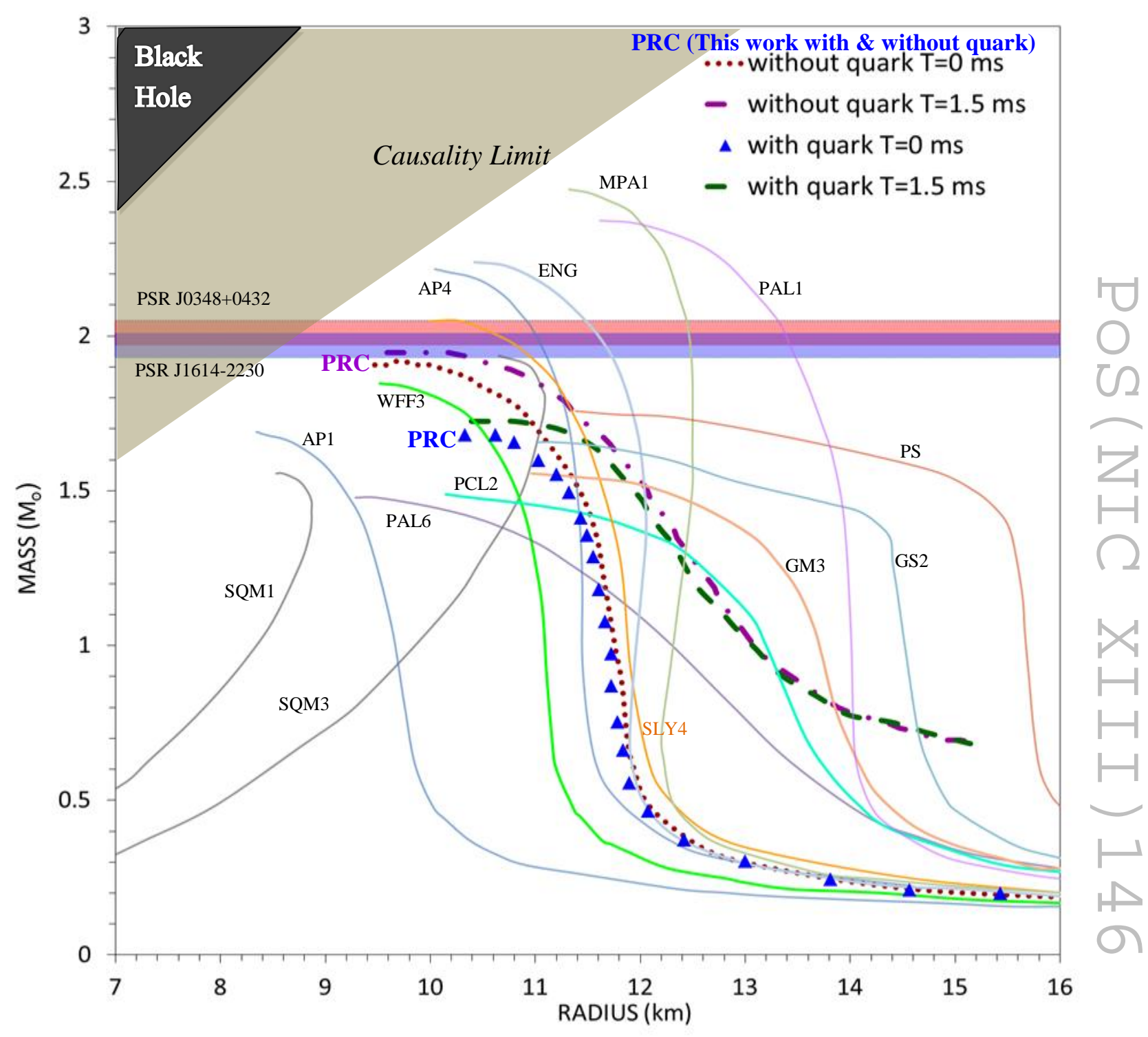

FIG.2 Present calculations for Mass-Radius relationship with and without presence of quark matter inside the core. for static $(T=0 \mathrm{~ms})$ neutron stars are shown by two curves 'PRC' and calculations for rotating neutron stars $(T=1.5 \mathrm{~ms})$ with and without quark are shown by another two curves 'PRC'. M-R relationship predicted by many other models are also shown.

It is obvious that the presence of quarks as constituents of stellar core significantly affects the mass-radius relationship for massive stars with smaller radii. The stars with relatively lower masses and higher radii are almost unaffected by its constituents. On the contrary, the effect of 
rotation becomes more prominent in lower mass region of the curves presenting the mass-radius relationship for the neutron stars with and without quark.

\section{Summary}

The recent observations of the binary millisecond pulsar J1614-2230 by P.B. Demorest et al. [4] and PSR J0348+0432 by J. Antoniadis et al. [5] suggest that the masses lie within $1.97 \pm$ $0.04 \mathrm{M}_{\odot}$ and $2.01 \pm 0.04 \mathrm{M}_{\odot}$, respectively, where $\mathrm{M}_{\odot}$ is the solar mass. In conformity with recent observations, a pure nucleonic EoS determines that the maximum mass of NS rotating with frequency $v \sim 667 \mathrm{~Hz}$ below r-mode instability is $\sim 1.95 \mathrm{M}_{\odot}$ with radius $\sim 10 \mathrm{~km}$. However, the maximum mass of NS with quark cores rotating with the maximum frequency limited by the r-mode instability i.e. $v \sim 667 \mathrm{~Hz}$, reduces to $\sim 1.72 \mathrm{M}_{\odot}$ only. Although the present models do not support the massive compact star, the existence of deconfined quarks inside stellar core can not be ruled out. The issue of quark matter in NS is not fully resolved yet and needs to be reconsidered with improved models for the high-density equation of state.

This work was financially supported in part by the University Grant Commission of India under UGC Foreign Travel Grant F.No.6-195/2014(TG).

\section{References}

1. N. K. Glendenning, Compact Stars, Nuclear Physics, Particle Physics, and General Relativity, 2nd edn. (Springer-Verlag, 2000).

2. F. Weber, Prog. Part. Nucl. Phys. 54, 193 (2005).

3. D. Blaschke, N. K. Glendenning and A. Sedrakian, Physics of Neutron Star Interiors, Lecture Notes in Physics, Vol. 578 (Springer, 2001).

4. P.B. Demorest, T.Pennucci, S.M.Ransom, M.S.E. Roberts, J.W.T. Hessels, Nature 467, 1081 (2010)

5. J. Antoniadis et al., Science 340, 1233232 (2013).

6. F. Weber, G.A. Contrera, M.G. Orsaria, W. Spinella and O. Zubairi, Mod. Phys. Lett. A 29, $1430022(2014)$

7. D.H.Wen, J. Yan and X.M. Liu, Int. J. Mod. Phys. D 21, 1250036 (2012)

8. N. Stergioulas, J.L. Friedman, Astrophys. J. 444, 306 (1995).

9. Partha Roy Chowdhury et al; Phys. Rev. C 81, (2010) 062801(R).

10. A. Kurkela, P. Romatschke, A. Vuorinen, Phys. Rev. D 81, 105021 (2010).

11. A. Chodos, R. L. Jaffe, K. Johnson and C. B. Thorn, Phys. Rev. D 10, 2599 (1974).

12. Partha Roy Chowdhury, "Nuclear matter... properties", PoS (NIC XI) 175 (2010)

(Proc.Int. Symp. NIC XI, Heidelberg, Germany, 19-23 July, 2010).

13. James M. Lattimer, Annu.Rev.Nucl.Part.Sci. 62, 485 (2012) arXiv:1305.3510v1 [nucl-th]

14. J. M. Lattimer and M. Prakash, Astrophys. J. 550, 426 (2001). 